Nota / Note

\title{
PLANTAS VISITADAS POR ABELHAS AFRICANIZADAS EM DUAS LOCALIDADES DO ESTADO DE SÃO PAULO
}

\author{
Luís Carlos Marchini" ${ }^{1 *}$; Augusta Carolina de Camargo Carmello Moreti ${ }^{2,4}$; Erica Weinstein \\ Teixeira ${ }^{2}$; Etelvina Conceição Almeida da Silva²; Ricardo Ribeiro Rodrigues³; Vinicius Castro \\ Souza ${ }^{3}$ \\ ${ }^{1}$ Depto. de Entomologia, Fitopatologia e Zoologia Agrícola - USP/ESALQ, C.P. 9 - CEP: 13418-900 - Piracicaba, SP. \\ Instituto de Zootecnia/ SAA - C.P. 60 - CEP: 13460-000 - Nova Odessa, SP. \\ ${ }^{3}$ Depto. de Ciências Biológicas - USP/ESALQ. \\ ${ }^{4}$ Bolsista CNPq. \\ *Autor correspondente <lcmarchi@carpa.ciagri.usp.br>
}

RESUMO: O conhecimento das plantas de uma região, sua época de florescimento e as características do pólen, auxiliam na determinação das espécies vegetais que contribuem para a composição do mel. Durante o período de 1994 a 1997 foram inventariadas, semanalmente, em diferentes horários e num raio de aproximadamente $300 \mathrm{~m}$ dos apiários da USP/ESALQ, em Piracicaba,SP ( $22^{\circ} 43^{\prime} \mathrm{S}, 47^{\circ} 27^{\prime} \mathrm{W}$ e $580 \mathrm{~m}$ ) e do Instituto de Zootecnia, em Pindamonhangaba, SP $\left(22^{\circ} 57^{\prime} \mathrm{S}, 45^{\circ} 27^{\prime} \mathrm{W}\right.$ e $\left.560 \mathrm{~m}\right)$, as plantas que estavam sendo visitadas por abelhas. Foram anotados o mês e o período de florescimento das plantas inventariadas. Em Piracicaba foram identificadas 94 espécies de plantas pertencentes a 41 famílias (maior número de espécies nas famílias Asteraceae e Myrtaceae) e em Pindamonhangaba, 76 espécies, pertencentes a 26 famílias (maior número de espécies nas famílias Asteraceae e Verbenaceae). Os resultados indicam que 21 famílias, 22 gêneros e 15 espécies de plantas visitadas pelas abelhas africanizadas foram comuns em ambas as localidades.

Palavras-chave: Apis mellifera, abelha, recurso floral, similaridade

\section{PLANTS VISITED BY AFRICANIZED HONEY BEES IN TWO LOCALITES OF THE STATE OF SÃO PAULO}

\begin{abstract}
The knowledge of plants from a given region, as well as the blooming period and the pollen characteristics, help for the determination of the plant species which contribute for the honey composition. Plants visited by honey bees were listed during the period 1994 to 1997 . The survey was carried out weekly, at different times and within a $300 \mathrm{~m}$ radius at two sites: the apiaries of USP/ESALQ in Piracicaba, SP $\left(22^{\circ} 43^{\prime} \mathrm{S}\right.$, $47^{\circ} 27^{\prime} \mathrm{W}$ e $\left.580 \mathrm{~m}\right)$ and of the 'Instituto de Zootecnia', in Pindamonhangaba, SP (22 $57^{\prime} \mathrm{S}, 45^{\circ} 27^{\prime} \mathrm{W}$ e $\left.560 \mathrm{~m}\right)$, both in Brazil. The month and the blooming period were recorded. A total of 94 plants species, from 41 families was identified in Piracicaba (the largest number of species in Asteraceae and Myrtaceae) and 76 plant species of 26 families, in Pindamonhangaba (the largest number of Asteraceae and Verbenaceae). The results indicated that 21 families, 22 genera and 15 species were common for both localities.

Key words: Apis mellifera, honey bee, floral source, similarity
\end{abstract}

\section{INTRODUÇÃO}

A coleta e a identificação taxonômica de espécies que compõem a fauna e a flora de uma determinada região são importantes para o conhecimento dos recursos naturais nela disponíveis, possibilitando a obtenção de informações básicas para estudos mais amplos sobre as características ecológicas de um determinado habitat ou ecossistema (Prado, 1980). Por outro lado, o conhecimento detalhado das plantas que florescem numa determinada região, sua época de florescimento e as características do pólen podem auxiliar na determinação das espécies vegetais que contribuíram para a composição final do mel produzido naquela região (Santos, 1964).

No Brasil, os primeiros levantamentos relacionando abelhas e flores foram realizados, no estado do Pará, por Ducke (1902; 1906), citados por Viana (1992), contudo, somente a partir da década de 60 surgiram os levantamentos sistematizados, com metodologia padronizada (Sakagami et al., 1967). Ramalho et al. (1990) apresentaram um extenso artigo de revisão sobre as plantas importantes para abelhas Melipona, Trigonini e para abelhas africanizadas em habitats neotropicais no qual incluem basicamente todos os trabalhos, realizados em diferentes regiões brasileiras, sobre levantamento de plantas utilizadas por abelhas, de diferentes espécies, para coleta de pólen e néctar.

Mais recentemente têm sido desenvolvidos trabalhos, amostrando, principalmente, as plantas apícolas e as abelhas silvestres que as visitam, como os de Martins (1990; 1994) e Castro (1994), nas 
caatingas da Bahia, Pedro (1992) em área de cerrado do Estado de São Paulo, Cure et al. (1993) em Viçosa, MG, Aguiar et al. (1995) em área de caatinga do cariri paraibano, Marques-Souza (1995), na Amazônia Central, Gonçalves et al. (1996) em mata secundária em Alcântara, MA, Carvalho \& Bego (1997) na reserva ecológica do Panga, Uberlândia, MG, Carvalho et. al. (1998), no campus da ESALQ, em Piracicaba, Estado de São Paulo, Mateus (1998) em Luiz Antônio, Estado de São Paulo, Carvalho (1999), em área de caatinga, no município de Castro Alves, Estado da Bahia, entre muitos outros.

$O$ presente trabalho foi desenvolvido com o objetivo de inventariar as plantas visitadas por abelhas africanizadas (Apis mellifera L.) em duas localidades do Estado de São Paulo, comparando as plantas utilizadas como fonte de alimento pelas abelhas em cada uma destas localidades, verificando suas semelhanças.

\section{MATERIAL E MÉTODOS}

De 1994 a 1997 foram inventariadas as plantas que estavam florescendo, e sendo visitadas por abelhas Apis mellifera $\mathrm{L}$., num raio de aproximadamente $300 \mathrm{~m}$ dos apiários do Departamento de Entomologia da USP/ ESALQ, Campus de Piracicaba, e do Instituto de Zootecnia, em Pindamonhangaba, SP. Nos dois locais existem resquícios da mata natural, plantas ornamentais e de interesse apícola introduzidas ao longo dos anos e outras plantas consideradas como invasoras.

As coletas foram realizadas semanalmente, em dias secos e em diferentes horários. As plantas foram preparadas em estrados ou pranchas de madeira, entre folhas de jornal, fazendo com que folhas e frutos ficassem bem distribuídos. O material depois de seco em estufa a $55^{\circ} \mathrm{C}$ por 48 horas, foi encaminhado ao Departamento de Botânica da ESALQ/ USP, para identificação. Foram anotados o mês e a duração do florescimento das plantas inventariadas.

Com os resultados dos levantamentos realizados nas localidades Piracicaba, SP(I) e Pindamonhangaba, SP (II) calculou-se o coeficiente de similaridade de Sorensen (Krebs, 1989) cuja fórmula é a seguinte:

$$
S=2 a /(2 a+b+c) C 100,
$$

Onde, $a=$ número de famílias ou gêneros ou espécies de plantas comuns às duas localidades (I e II); $b=$ número de famílias ou gêneros ou espécies de plantas que ocorre exclusivamente na localidade I; c= número de famílias ou gêneros ou espécies de plantas que ocorre exclusivamente na localidade II.

O Coeficiente de Sorensen pode variar de $0 \%$, quando não há nenhuma coincidência entre as áreas amostradas, até $100 \%$, quando a coincidência é total.

Utilizando o mesmo coeficiente de similaridade, foram comparados os dados obtidos por Santos (1961) (I) e os resultados da presente pesquisa (II) pois ambos os trabalhos foram realizados no mesmo local, campus da USP/ESALQ, em Piracicaba, SP.

\section{RESULTADOS E DISCUSSÃO}

Em Piracicaba, SP, foram identificadas 94 espécies de plantas (TABELA 1), pertencentes a 41 famílias: Acanthaceae (1 espécie), Amaranthaceae (2 espécies), Anacardiaceae (2 espécies), Apiaceae (1 espécie), Apocynaceae (2 espécies), Arecaceae (1 espécie), Asteraceae (9 espécies), Balsaminaceae (1 espécie), Bignoniaceae (3 espécies), Bombacaceae (2 espécies), Boraginaceae (1 espécie), Caesalpiniaceae (4 espécies), Caricaceae (1 espécie), Combretaceae (1 espécie), Commelinaceae (1 espécie), Convulvulaceae (2 espécies), Cucurbitaceae (1 espécie), Erythroxylaceae (1 espécie), Euphorbiaceae (3 espécies), Fabaceae (3 espécies), Iridaceae (1 espécie), Lamiaceae (2 espécies), Lauraceae (1 espécie), Malpighiaceae (1 espécie), Malvaceae (4 espécies), Melastomataceae (2 espécies), Mimosaceae (5 espécies), Moraceae (1 espécie), Myrtaceae (8 espécies), Oxalidaceae (1 espécie), Poaceae (6 espécies), Polygonaceae (1 espécie), Rhamnaceae (2 espécies), Rubiaceae (1 espécie), Rutaceae (4 espécies), Sapindaceae (1 espécie), Solanaceae (4 espécies), Sterculiaceae (3 espécies), Tiliaceae (2 espécies), Ulmaceae (1 espécie), Verbenaceae (1 espécie).

Em Pindamonhangaba, SP, foram identificadas 76 espécies de plantas (TABELA 2), pertencentes a 26 famílias: Acanthaceae (1 espécie), Agavaceae (2 espécies), Asteraceae (15 espécies), Bignoniaceae (2 espécies), Boraginaceae (1 espécie), Brassicaceae (2 espécies), Caesalpiniaceae (5 espécies), Convolvulaceae (2 espécies), Cucurbitaceae (1 espécie), Ericaceae (1 espécie), Fabaceae (3 espécies), Lamiaceae (2 espécies), Lauraceae (1 espécie), Malvaceae (2 espécies), Melastomataceae (3 espécies), Mimosaceae (6 espécies), Myrtaceae (5 espécies), Oleaceae (3 espécies), Pedaliaceae (1 espécie), Poaceae (4 espécies), Polygonaceae (1 espécie), Rhamnaceae (1 espécie), Rubiaceae (2 espécies), Sapindaceae (1 espécie), Sterculiaceae (2 espécies) e Verbenaceae (7 espécies).

Comparando as duas localidades foram coincidentes 21 famílias, 22 gêneros e 15 espécies de plantas visitadas por abelhas africanizadas.

Segundo Ramalho et al. (1990), as famílias Leguminosae, Myrtaceae, Palmae e Rubiaceae, são as mais importantes para as abelhas africanizadas, Trigonini e Melipona e, segundo Pirani \& Cortopassi-Laurino (1993), as famílias mais visitadas por diversas espécies de abelhas sociais, incluindo as abelhas africanizadas, foram Compositae (24 espécies) (hoje classificadas como Asteraceae) e Leguminosae (21 espécies) (hoje Caesalpiniaceae, Fabaceae e Mimosaceae). Comparando os resultados do presente trabalho com os citados anteriormente, pode-se verificar que a maioria das famílias de plantas listadas como importantes para as abelhas africanizadas, por Ramalho et al. (1990) e Pirani \& Cortopassi-Laurino (1993), e ainda, Pereira (1994), foram aqui amostradas. 
Os coeficientes de similaridade calculados comparando as duas localidades amostradas foram $\mathrm{S}=62,68 \%$; $\mathrm{S}=31,43 \%$ e $\mathrm{S}=17,64 \%$ para família, gênero e espécies de plantas, respectivamente. Para gêneros, o índice de similaridade no presente trabalho, encontrase dentro da faixa observada por Miranda \& Carneiro Filho (1994) quando comparam sete savanas amazônicas, duas a duas, obtendo uma variação entre as diferentes áreas de 29 a $54 \%$.

Considerando que o primeiro levantamento de plantas, visando a identificação do pólen contido no mel, foi realizado por Santos (1961) no campus da ESALQ em Piracicaba, SP, nos meses de junho a outubro, foram comparados os dados por ele obtidos com os observados no presente trabalho. Pode-se verificar que 21 famílias, 24 gêneros e 17 espécies de plantas foram coincidentes, apresentando coeficientes de similaridade de: $S=59,15 \%$; $S=30,38 \%$ e $S=19,64 \%$ para família, gênero e espécies de plantas, respectivamente.

Nas TABELAS 1 e 2 podem, ainda, ser observados os calendários de florescimento das plantas visitadas por abelhas nas duas localidades amostradas, sendo estas informações úteis tanto no estabelecimento de apiários como na manutenção e manejo dos mesmos.

TABELA 1 - Calendário de florescimento das plantas de interesse apícola, inventariadas no Campus da ESALQ/USP, em Piracicaba, SP, no período de 1994 a 1997.

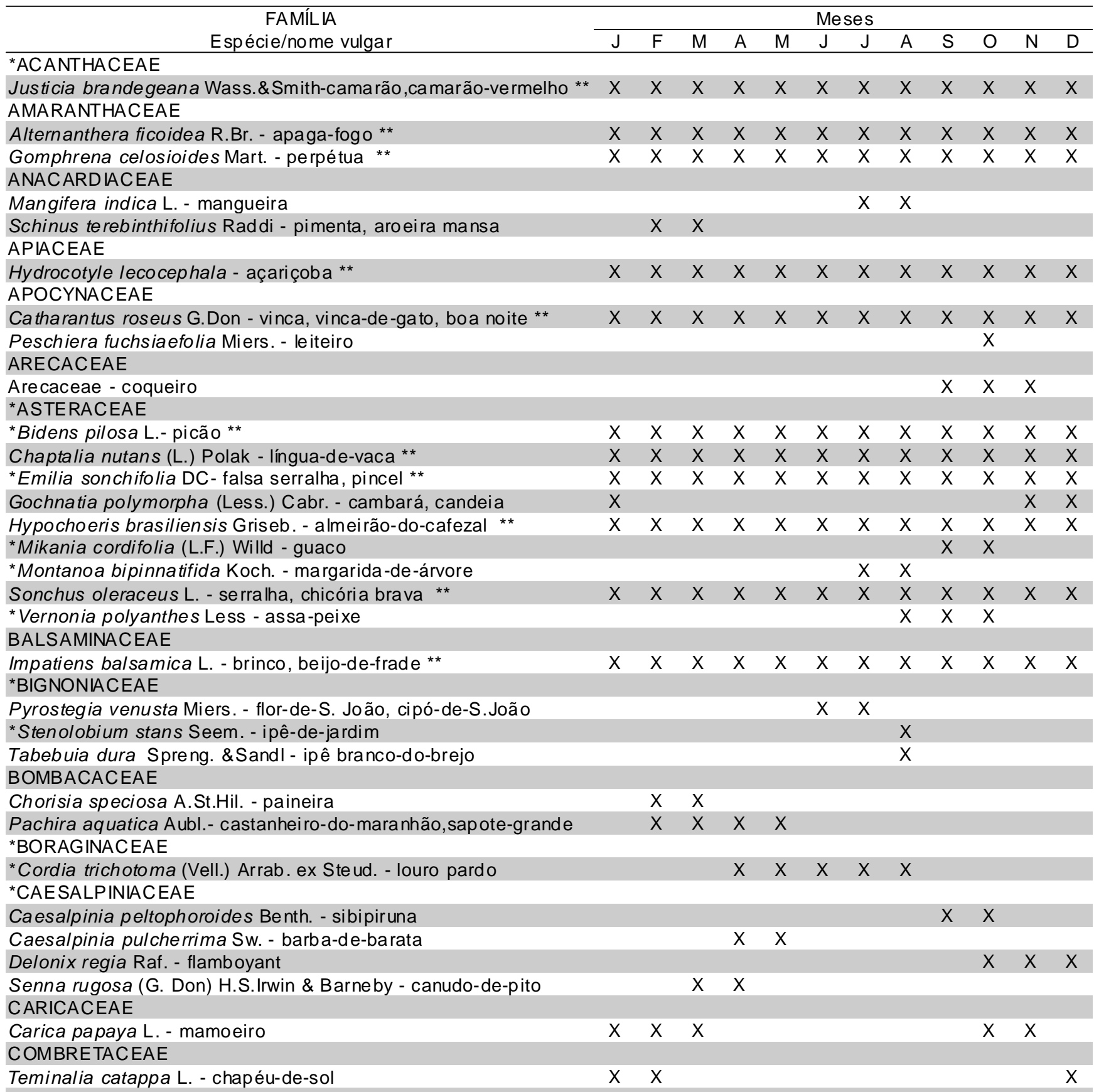


Tradescantia elongata Meyer - trapoeiraba ** ${ }^{*}$ CONVOLVULACEAE

Ipomoea cairica (L.) Sweet. - corda-de-viola **

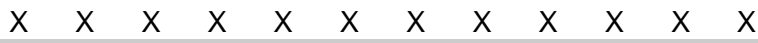
Jacquemontia sp. - corda-de-viola ** ${ }^{*}$ CUCURBITACEAE

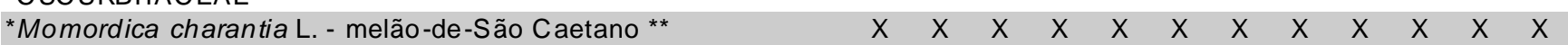
ERYTHROXYLACEAE

Erythroxylum buxux Peyr - buchinho

EUPHORB IACEAE

Croton urucurana Baill. - sangra d'água

Euphorbia pulcherrima Wild. - bico-de-papagaio

Phyllanthus corcovadensis Muell. - quebra-pedra **

*FABACEAE

Crotalaria incana L. - xique-xique, gerge lim bravo

Macroptilium sp. - macropitilo

Tipuana tipo (Benth.)Kuntze - tipuana

$\begin{array}{lllllllllllllll}x & x & x & x & x & x & x & x & x & x & x & x\end{array}$

IRIDACEAE

Belamcanda chinensis D.C. - flor leopardo

*LAMIACEAE

Leonurus japonicus Ho utt. - rubim **

Salvia splendens Ker-Gawl. - salvia

*LAURACEAE

${ }^{*}$ Persea americana Mill - abacateiro

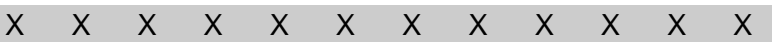

MALPIGHIACEAE

Lophantera lactescens Ducke- chuva-de-ouro

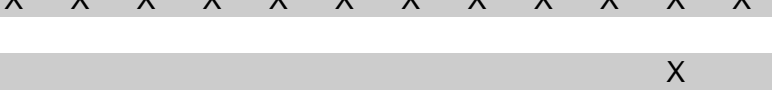

*MALVACEAE

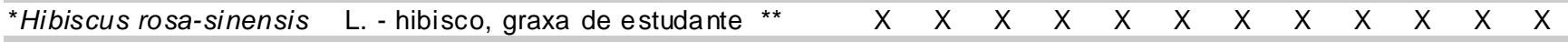

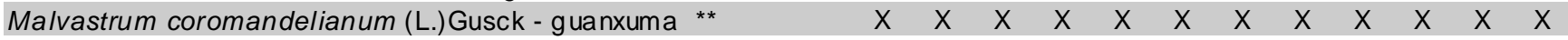

Malvaviscus arboreus Cav. -malvavisvo **

Wissadula subpeltata (Kuntze)Fries - malva-estrela **

*MELASTOMATACEAE

Tibouchina granulosa Cogn - quaresmeira

Tibouchina stenocarpa(Schrank \& Mart. ex DC.)Cogn.

*MIMOSACEAE

Inga fagifolia Willd. - ingá

Leucaena leucocephala (Lam.) de Wit- leucena

Mimosa caesalpinifolia Baenth.- sabiá, sansão-do-campo

Mimosa bimucronata (DC.)O.Kuntze - maricá

Mimosaceae

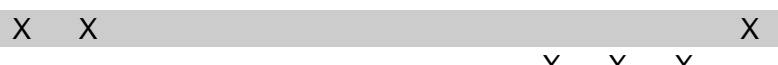

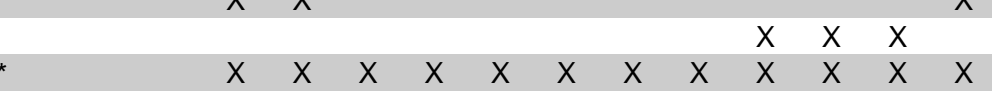

$\begin{array}{lll}X & X & X \\ X & x & x\end{array}$

MORACEAE

Morus nigra L. - amoreira

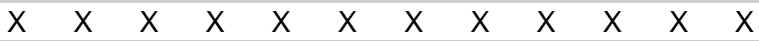

*MYRTACEAE

*Eucalyptus sp. - eucalipto

*Eucalyptus sp. - eucalipto

Eucalyptus saligna Sm.- eucalipto

Eugenia involucrata DC.- cerejeira-do-mato

Eugenia uvalha Camb. - uvaia

${ }^{*}$ Myrciaria cauliflora Mart. - jaboticabeira

Psidium guayava L. - goiabeira

Psidium sp. - goiabeira africana

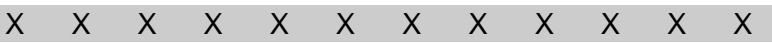

OXALIDACEAE

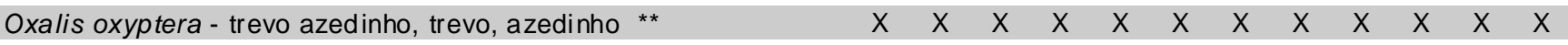

*POACEAE

Brachiaria sp. - braquiaria

Cynodon dactylon (L.)Pers. - grama seda **

Panicum maximum Jacq. - capim colonião **

Paspalum notatum Flugee- grama batatais **

$\mathrm{X} \times \mathrm{X}$

Setaria gemiculata (Lam.)Beauv. - bambuzinho **

Zea mays L. - milho

$\begin{array}{lllllllllllllll}X & X & X & X & X & X & X & X & X & X & X & X \\ \end{array}$

$\mathrm{X} \quad \mathrm{X}$

*POLYGONACEAE

Triplaris brasiliana Cham. - pau formiga, formigueiro

${ }^{*}$ RHAMNACEAE

*Hovenia dulcis Thumb. - uva japonesa

Rhamnidium elaecarpum Reissek - saquaraji amarelo

$\mathrm{X}$

$\begin{array}{lll}X & X & X \\ X & X & X \\ X & \end{array}$

$\mathrm{X} X$

$\mathrm{X}$

X $\quad X$

X $\mathrm{X}$

X

Scientia Agricola, v.58, n.2, p.413-420, abr./jun. 2001 
${ }^{*}$ RUBIACEAE

${ }^{*}$ Coffea arabica L. - cafe eiro

RUTACEAE

Citrus sp. - citros

$x \quad x$

Citrus sinensis (L.) Osbeck - Iaranjeira

$x \quad x$

Prunus persica Batsch - pessegueiro

$x \quad x$

Zanthoxylum chiloperone Mart. ex Engl.

$x \quad x$

*SAPINDACEAE

Allophyllus edulis (A.St. Hill) Radlk.

$\mathrm{X}$

SOLANACEAE

Acnistus arborescens (L.) Schtdl. - marianeira **

Solanum americanum Mill. - maria pretinha **

Solanum concinnum Schott ex Sendth. - joá velame **

Solanum erianthum D.Don - fumo bravo **

*STERCUL IACEAE

*Dombeya burgessiae Gerr. ex Harv. et Sond. - astrapéia

Dombeya spectabilis Bojer - astrapéia

*Dombeya wallichii Daydon Jackson -astrapéia rosa

TILIACEAE

Corchorus hirtus L. **

Luehea divaricata Mart. et Zucc. - açoita cavalo

ULMACEAE

Trema micrantha (L.) Blume - crindiúva

*VERBENACEAE

Aloysia virgata (Ruiz et Pav.) A.L.Juss. - lixa, lixeira

$\mathrm{X} X$

${ }^{*}$ Famílias e espécies de plantas comuns às duas localidades avaliadas.

${ }^{* *}$ Não florescem com a mesma intensidade o ano todo.

***O florescimento depende da época de plantio

TABELA 2 - Calendário de florescimento das plantas de interesse apícola, inventariadas no Centro de Apicultura Tropical do Instituto de Zootecnia, em Pindamonhangaba, SP, no período de 1994 a 1997.

\begin{tabular}{|c|c|c|c|c|c|c|c|c|c|c|c|c|}
\hline \multirow{2}{*}{$\begin{array}{c}\text { FAMÍLIA } \\
\text { Espécie/ no me vulgar }\end{array}$} & \multicolumn{12}{|c|}{ Meses } \\
\hline & $J$ & $\mathrm{~F}$ & M & A & M & $\mathrm{J}$ & $\mathrm{J}$ & A & S & $\mathrm{O}$ & $\mathrm{N}$ & $\mathrm{D}$ \\
\hline \multicolumn{13}{|l|}{${ }^{*} \mathrm{ACANTHACEAE}$} \\
\hline Pachystachys luthea Nees - camarão amarelo & & & $\mathrm{X}$ & $X$ & & & & & & & & \\
\hline \multicolumn{13}{|l|}{ AGAVACEAE } \\
\hline Cordilyne terminalis Kunth. - dracena vermelha & & & & $X$ & $X$ & $X$ & & & & & & \\
\hline Dracena fragans Ker.Gawl. - dracena & & & & & $\mathrm{X}$ & $\mathrm{X}$ & $\mathrm{X}$ & & & & & \\
\hline \multicolumn{13}{|l|}{ *ASTERACEAE } \\
\hline Baccharis semiserrata DC. - alecrim-do-campo & $X$ & $X$ & & & & & & & & & & \\
\hline${ }^{*}$ Bidens pilosa L.- picão ** & $\mathrm{X}$ & $X$ & $X$ & $X$ & $X$ & $X$ & $X$ & $X$ & $X$ & $X$ & $X$ & $\mathrm{X}$ \\
\hline Bidens sulphuria Sch.Big.- cosmos ** & $\mathrm{X}$ & $\mathrm{X}$ & $\mathrm{X}$ & $\mathrm{X}$ & $\mathrm{X}$ & $\mathrm{X}$ & $\mathrm{X}$ & $\mathrm{X}$ & $\mathrm{X}$ & $\mathrm{X}$ & $\mathrm{X}$ & $\mathrm{X}$ \\
\hline${ }^{*}$ Emilia sonchifolia DC - falsa serralha, pincel ${ }^{\text {** }}$ & $\mathrm{X}$ & $X$ & $\mathrm{X}$ & $\mathrm{X}$ & $\mathrm{X}$ & $\mathrm{X}$ & $\mathrm{X}$ & $\mathrm{X}$ & $\mathrm{X}$ & $\mathrm{X}$ & $\mathrm{X}$ & $\mathrm{X}$ \\
\hline Eupathorium sp. & & & & & & & & & $\mathrm{X}$ & $X$ & & \\
\hline Eupathorium maximiliani Schrad. & & & $\mathrm{X}$ & $\mathrm{X}$ & $\mathrm{X}$ & & & & & & & \\
\hline *Mikania cordifolia (L.F.) Willd - guaco & & & & & & $X$ & $X$ & $X$ & & & & \\
\hline *Montanoa bipinnatifida Koch. - margarida-de-árvore & & & & & $X$ & $\mathrm{X}$ & $\mathrm{X}$ & & & & & \\
\hline Senecio brasiliensis Less - flor-das-almas, maria mole & & & & & & & & & & $X$ & $X$ & \\
\hline Thithonia diversifolia A. Gray - margaridão amarelo & & & & & & & & $X$ & $\mathrm{X}$ & $\mathrm{X}$ & & \\
\hline Vernonia sp. - cambará-do-brejo & & & & & $\mathrm{X}$ & $\mathrm{X}$ & & & & & & \\
\hline Vernonia cognata (Chod.) Cabrl & & & & & & $\mathrm{X}$ & $X$ & $X$ & & & & \\
\hline *Vernonia polyanthes Less. - assa-peixe & & & & & & $X$ & $\mathrm{X}$ & & & & & \\
\hline Vernonia scorpioides (Lam.) Pears. - erva preá & & & & & & $\mathrm{X}$ & $\mathrm{X}$ & $\mathrm{X}$ & & & & \\
\hline Zinnia elegans Jacq. - capitão-do-campo ** & $\mathrm{X}$ & $\mathrm{X}$ & X & $\mathrm{X}$ & $\mathrm{X}$ & $\mathrm{X}$ & $\mathrm{X}$ & $\mathrm{X}$ & $\mathrm{X}$ & $\mathrm{X}$ & $\mathrm{X}$ & X \\
\hline \multicolumn{13}{|l|}{ *BIGNONIACEAE } \\
\hline Arvabideae sp. & & & & $\mathrm{X}$ & $\mathrm{X}$ & & & & & & & \\
\hline *Stenolobium stans Seem. - ipê-de-jardim & & & & & & & & $X$ & & & & \\
\hline
\end{tabular}

*BORAGINACEAE

Scientia Agricola, v.58, n.2, p.413-420, abr./jun. 2001 
*Cordia trichotoma (Vell.) Arrab. ex Steud.- louro pardo

$\mathrm{X} \times \mathrm{X}$

BRASSICACEAE

Raphanus raphanistrum L. - nabiça

Raphanus sativus L. - rabanete

${ }^{*}$ CAESALPINIACEAE

Bauhinia variegata L. - casco- de- vaca

Cassia ferruginea (Schrad.) Schrad. ex DC - chuva-de-ouro

Cassia fistula L. - cassia imperial, brinco-de-princesa.

Senna sp.

Senna bicapsularis (L.) Roxb. - cassia

${ }^{*}$ CONVOLVULACEAE

Evolvulus sp.

Ipomoea hederifolia L. - corda-de-viola **

*CUCURBITACEAE

*Momordica charantia L. - melão-de-São Caetano **

ERICACEAE

Rhododendron simsii Planch - azaléia

*FABACEAE

Cajanus cajan Millsp - feijão guandu

Crotalaria spectabilis Moth - crotalaria

Glycine max Merril - soja

${ }^{*}$ LAMIACEAE

Ocimum micranthum - manjericão

Tetrade nia riparia (Hochst.) Codd. - mirra

*LAURACEAE

*Persea americana L. - abacateiro

*MALVACEAE

${ }^{*}$ Hibiscus rosa-sinensis L.- hibisco, graxa-de-estudante

Hibiscus schizopetalis - hibisco retorcido

*MELASTOMATACEAE

Miconia ligustroides (DC.) Naud. - jacatirão

Tibouchina gracilis Cogn - quaresmeira

Trembleya parviflora (D.Don) Cogn.

*MIMOSACEAE

Anadenanthera colubrina (Vell.)Brenan - angico

Calliandra foliolosa Benth. - diadema **

Calliandra tweedii Benth. - esponjeira, caliandra vermelha**

Enterolobium cortortisiliquim(Vell.) Morongi-ore lha-de-negro **

Mimosa bimucronata (DC.) O. Kuntze - maricá

Mimosa pudica K. - sensitiva

${ }^{*}$ MYRTACEAE

${ }^{*}$ Eucalyptus sp. - eucalipto.

Eugenia pyriformis. - orvalha

Eugenia uniflora L. - pitangueira

Myrcia sp - guamirim branco

${ }^{*}$ Myrciaria cauliflora Mart. - jaboticabeira

OLEACEAE

Ligustrum indicum Merr. - ligustro

Ligustrum lucidum Ait. ligustro

Ligustrum sinense Lour. - alfineteiro-do-japão

PEDALACEAE

Sesamum indicum $\mathrm{L}$ - gergelim

*POACEAE

Avena sativa L. - aveia

Bambusa dissimulator - bambu

Brachiaria sp. - braquiária **

Triticum aestivum L. - trigo

*POLYGONACEAE

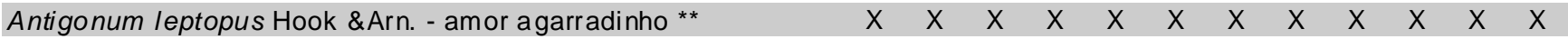

${ }^{*}$ RHAMNAC EAE

${ }^{*}$ Hovenia dulcis Thumb. - uva japonesa

${ }^{*}$ RUBIACEAE

Borreria sp. - carrapicho

${ }^{*}$ Coffea arabica L. - cafe eiro

$\begin{array}{llllllllllll}X & X & X & X & X & X & X & X & X & X & X & X \\ X & X & X & X & X & X & X & X & X & X & X & X \\ X & X & & & & & & & & X & X & X \\ X & & & & & & & & & & & X \\ & & & & & & & & & X & X\end{array}$

$\begin{array}{llllllllllll}X & X & X & X & X & X & X & X & X & X & X & X \\ X & X & X & X & X & X & X & X & X & X & X & X\end{array}$

$\mathrm{X}$

$\begin{array}{lll}X & X & X \\ X & X & x\end{array}$

$x \quad x$ $\mathrm{X} X$

Scientia Agricola, v.58, n.2, p.413-420, abr./jun. 2001 


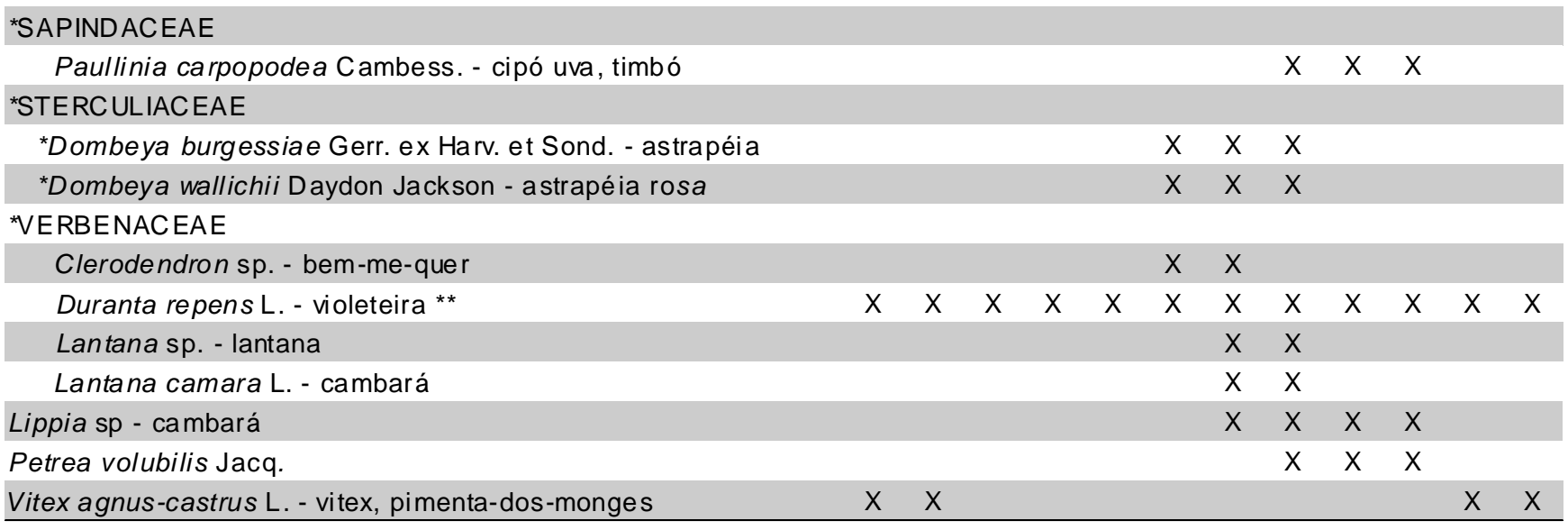

*Famílias e espécies de plantas comuns às duas localidades avaliadas.

**Não florescem com a mesma intensidade o ano todo.

${ }^{* * *}$ Florescimento depende da época de plantio

\section{CONCLUSÕES}

As duas localidades apresentam poucas semelhanças com referência a flora de interesse apícola uma vez que apenas $17,64 \%$ das espécies são coincidentes.

As informações referentes ao calendário de florescimento das plantas de interesse apícola para as duas localidades estudadas podem ser utilizadas tanto no estabelecimento de apiários como na manutenção e manejo dos mesmos.

\section{AGRADECIMENTO}

À Fundação de Amparo à Pesquisa do Estado de São Paulo (FAPESP), pelo financiamento parcial da presente pesquisa (Processo 1995/3222-4).

\section{REFERÊNCIAS BIBLIOGRÁFICAS}

AGUIAR, C.M.L.; MARTINS, C.F.; MOURA, A.C. de A. Recursos florais utilizados por abelhas silvestres (Apoidea) na caatinga de São João do Cariri, Paraíba. Revista Nordestina de Biologia, v.10, p.101-117, 1995.

CARVALHO, A.M.C.; BEGO, L.R. Exploitation of available resources by bee fauna (Apoidea-Hymenoptera in the Reserva Ecológica do Panga, Uberlândia, Satate of Minas Gerais, Brazil. Revista Brasileira de Entomologia, v.41, p.101-107, 1997.

CARVALHO, C.A.L. Diversidade de abelhas (Hymenoptera, Apoidea) e plantas visitadas no Município de Castro Alves, BA. Piracicaba, 1999. 104p. Tese (Doutorado) - Escola Superior de Agricultura "Luiz de Queiroz", Universidade de São Paulo.

CARVALHO, C.A.L.; MARCHINI, L.C.; BOS, P.B. Fontes de pólen utilizadas por Apis mellifera e Meliponinae (Hymenoptera; Apidae). In: ENCONTRO SOBRE ABELHAS, 3., Ribeirão Preto, 1998. Anais. Ribeirão Preto: FFCL/USP, 1998, p.258.

CASTRO, M.S. Composição, fenologia e visita às flores pelas espécies de Apidae em um ecossistema de caatinga (Nova Casa Nova- 9²6'S/ 4150'W). São Paulo, 1994. 103p. Dissertação (Mestrado) - Instituto de Biociências, Universidade de São Paulo.
CURE, J.R.; BASTOS FILHO, G.S.; OLIVEIRA, M.J.F. de; SILVEIRA, F.A. da. Levantamento de abelhas silvestres na zona da mata de Minas Gerais: I. Pastagem na Região de Viçosa (Hymenoptera, Apoidea). Revista Ceres, v.40, p.130-161, 1993.

GONÇALVES, S. de J.M.; RÊGO, M.; ARAÚJO, A. de. Abelhas sociais (Hymenoptera: Apidae) e seus recursos florais em uma região de mata secundária, Alcântara, MA, Brasil. Acta Amazonica, v.26, p.55-68, 1996.

KREBS, C.J. Ecological methodology. New York: Harper Collins Publishers, 1989. 654p.

MARQUES-SOUZA, A.C. Fontes de pólen exploradas por Melipona compressipes manaosensis (Apidae: Meliponinae), abelha da Amazônia Central). Acta Amazonica, v.21, p.77-86, 1995.

MARTINS, C.F. Estrutura da comunidade de abelhas (Hymenoptera, Apoidea) na Caatinga(Casa Nova, BA) e na Chapada Diamantina (Lençóis, BA). São Paulo, 1990. 139p. Tese (Doutorado)- Instituto de Biociências, Universidade de São Paulo.

MARTINS, C.F. Comunidade de abelhas (Hymenoptera, Apoidea) da caatinga e do cerrado com elementos de campo rupestre do Estado da Bahia, Brasil. Revista Nordestina de Biologia, v.9, p.225-257, 1994.

MATEUS, S. Abundância relativa, fenologia e visita às flores pelos apoidea do cerrado da Estação Ecológica de Jatí, Luiz Antônio, SP. Ribeirão Preto, 1998. 159p. Dissertação (Mestrado) - Faculdade de Filosofia Ciências e Letras de Ribeirão Preto, Universidade de São Paulo.

MIRANDA, I.S.; CARNEIRO FILHO, A. Similaridade florística de algumas savanas Amazônicas. Boletim do Museu Paranaense Emílio Goeldi, Série Botânica, v.10, p.249-267, 1994.

PEDRO, S.R.M. Sobre as abelhas (Hymenoptera, Apoidea) em um ecossistema de cerrado (Cajuru, NE do Estado de São Paulo): composição, fenologia e visita a flores. Ribeirão Preto, 1992. 200p. Dissertação (Mestrado) - Faculdade de Filosofia, Ciências e Letras de Ribeirão Preto, Universidade de São Paulo.

PEREIRA, B.A. DA S. Flora apícola do cerrado. In: CONGRESSO BRASILEIRO DE APICULTURA,10., Pousada do Rio Quente, 1994. Anais. Pousada do Rio Quente: CBA, 1994. p.142-146.

PIRANI, J.R.; CORTOPASSI-LAURINO, M. (Coord.). Flores e abelhas em São Paulo. São Paulo: EDUSP, 1993. 192p.

PRADO, A.P. Importância prática da taxonomia: ou o papel da taxonomia para a entomologia aplicada). Revista Brasileira de Entomologia, v.24, p.165-167, 1980. 
RAMALHO, M.; KLEINERT-GIOVANNINI, A.; IMPERATRIZFONSECA, V.L. Important bee plants for stingless bees (Melipona and Trigonini) and Africanized honeybees (Apis mellifera) in neotropical habitats: a review. Apidologie, v.21, p.469-488, 1990.

SAKAGAMI, S.F.; LAROCA, S.; MOURE, J.S. Wild bees biocenotics in São José dos Pinhais (PR), South Brazil: preliminary report. Journal of the Faculty of Science Hokkaido University. Series VI, Zoology, v.16, p.253-291, 1967.

SANTOS, C.F.O. Avaliação do período de florescimento das plantas apícolas no ano de 1960, através do pólen contido nos méis e dos coletados pelas abelhas (Apis mellifera L.). Anais da ESALQ, v.21, p.253-264, 1964.
SANTOS, C.F.O. Morfologia e valor taxonômico do pólen das principais plantas apícolas. Piracicaba, 1961. 92p. Tese (Livre-Docência) - Escola Superior de Agricultura "Luiz de Queiroz", Universidade de São Paulo.

VIANA, B.F. Estudo da composição da fauna de Apidae e da flora apícola da Chapada Diamantina, Lençóis, Bahia $\left(12^{\circ}\right.$ 34'S/ 41L 23'W). São Paulo, 1992. 140p. Dissertação (Mestrado) - Instituto de Biociências, Universidade de São Paulo.

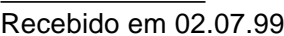

